\title{
Regression Analysis of Grid Stability under Decentralized Control
}

\author{
Deepak Kumar Panda, Saptarshi Das, Member, IEEE
}

\begin{abstract}
Decentralized smart grid control (DSGC) aims to implement smart control strategies through the demand response without significant reinforcements in the grid infrastructure. This strategy aims to balance the demand and supply considering the dynamic and economic price structure of the grid. Demand response strategy is implemented based on the heterogeneous nature of response of the consumers to the electricity price. In this paper, feature selection and regression analysis have been performed to study the dependence of the stability condition and system parameters on the parameters of decentralized control like the reaction time of the consumer, power produced or consumed and the price elasticity. The analysis is performed based on the data generated considering 10,000 Monte Carlo simulations of the initial conditions, which incorporates different characteristics of the heterogeneous consumers.
\end{abstract}

Keywords—power grid, stability, regression, feature selection

\section{INTRODUCTION}

The stability of the power grid depends on the balance between the power generation and demand. With the influx of uncontrollable renewable energy in the grid, demand response strategy is a promising solution [1]. With the increase in decentralized production of power, the flow of power is no longer unidirectional from the power producing companies to the consumers, instead it has become bidirectional. These changes in the power flow pattern make the consumers an attractive control center for maintaining the balance between energy demand and production.

There are many ways, which involve the consumers in smart grid control, aided by information technology and communication standards as defined in [2]. Decentralized control is preferable strategy as compared to centralized control as the former provides more robustness against cybersecurity and privacy [3], [4]. The indicator for the balance between demand and supply is reflected on the grid frequency fluctuations [5]. There have been studies about DSGC strategy as given in [6], [7] which involves response of the consumers based on the price fluctuation, hence playing an important role in grid stability. But as described in [8], the works done in [6], [7] have shortcomings in the form of fixed inputs issue and equality issue, where the former deals with changing only single parameter, hence limiting the input space of the remaining parameters. It also assumes no

The authors are with the Department of Mathematics, College of Engineering, Mathematics and Physical Sciences, University of Exeter, Penryn Campus, Cornwall TR10 9FE, U.K. (e-mail: dp457@exeter.ac.uk, saptarshi.das@ieee.org). interaction among the inputs parameters in determining the grid stability. While, the latter deals with equal input values which signifies equal willingness of the consumers to respond to the change in price signal. This violates the realistic nature of the operations in a major way. Data mining techniques are followed to classify the stability conditions based on the response of the heterogeneous consumers in [8], to delve deeper in the relationship between the parameters of the input space and the grid stability.

Several works have been done in the field of data mining for predicting the voltage [9], [10], angular stability [11]-[15] and blackouts [16]. Online transient stability prediction has been performed based on stream computing paradigm [11], which predicts the stability based on the rotor angle trajectories obtained from live synchro phasor data. Two class classification and data mining algorithm based on core vector machine (CVM) has been performed on the phasor measurement unit (PMU) big data in [12] for online transient stability assessment which makes it attractive to be used in real time. The stability problem of a high-dimensional power system has been solved using feature selection and support vector machine techniques in [13]. Transient stability boundary using the nonlinear transformation of the voltage magnitude and phase angles has been performed in [14] for the angular stability. Decision tree based out of step prediction of the generators has been performed in [15] for a series of operational and topological disturbances for the detection of the transient stability conditions and the algorithm was successfully tested on unseen samples. The voltage stability predictions using samples from the synchro-phasors are obtained using support vector machine (SVM) in [9] and active learning techniques has been used in [10], thus improving the system performance with lesser measurement samples. Improvement of the online learning speed and parameter tuning using extreme machine learning (ELM) has been performed in [16] for the detection of the blackouts. However, statistical relations of the stability index of the system has not been described in relation to demand response and price fluctuating index which is described in this paper with the help of regression and feature selection technique. Ordinary regression technique fits the parameters based on minimum least square technique while the Ridge regression penalizes the complexity of the model used for fitting the model. Bayesian regression aims to find the probabilistic model of the weights used in the regression problem to map the input parameters to the output space. Bayesian techniques 
for power grids has been applied in [17], [18] for load modelling and predicting the electricity demand, but these techniques are used in this paper to study the relationship of the input variables with the grid stability parameters.

The paper has been divided into sections where section II deals with the modelling used for decentralized control along with the assumptions with it. Section III describes the machine learning techniques involved with the modelling while Section IV and V discuss the simulation results, conclusion and scope of future work.

\section{SySTEM MODELING}

\section{A. Physical Model of the Synchronous Generator}

The synchronous generator model is derived from the energy conservation model of the generators and loads with its mathematical form similar to the Kuramoto oscillator model [19] and can be represented as:

$$
p_{j}^{\text {source }}=\frac{1}{2} M_{j} \frac{d}{d t}\left(\dot{\delta}_{j}^{2}\right)+\kappa\left(\dot{\delta}_{j}^{2}\right)-\sum_{k} P_{j k}^{\max } \sin \left(\delta_{k}-\delta_{j}\right),
$$

where, $j$ is the participant's index, $M$ is the moment of inertia, $\kappa$ is the friction coefficient and $P_{j k}^{\max }$ is the capacity of the line connecting the participant $j$ and $k$. As from the generator angle equation given in [6], we see that it can be represented as follows:

$$
\delta_{j}(t)=\omega t+\theta_{j}(t)
$$

where $\omega$ is the grid frequency and $\theta_{j}(t)$ is the rotor angle relative to it. Hence, substituting the value of $\delta_{j}(t)$ from (2) in the above equation yields:

$$
\frac{d^{2} \theta_{j}}{d t^{2}}=P_{j}-\alpha_{j} \frac{d \theta_{j}}{d t}+\sum_{k} K_{j k} \sin \left(\theta_{k}-\theta_{j}\right),
$$

where,

$$
K_{j k}=\frac{P_{j k}^{\max }}{M_{j} \omega}, \quad \alpha_{j}=\frac{2 \kappa_{j}}{M_{j}}, \quad P_{j}=\left[P_{j}^{\text {source }}-\kappa_{j} \omega^{2}\right] / M_{j} .
$$

As per [19], the model in equation (3) is correct based on the following assumptions:

$$
\frac{d \theta_{j}}{d t} \ll \omega, \quad \frac{d^{2} \theta_{j}}{d t^{2}} \ll \frac{2 \kappa_{j} \omega}{M_{j}} .
$$

\section{B. Price Structure Model}

The strategy for DGSC aims at encouraging the consumers to lower and increase their power consumption during high and lighter load respectively, and this is done with the help of a linear-price frequency fluctuation coefficient $p_{i}\left(\frac{d \theta}{d t}\right)$, which can be represented as follows:

$$
p_{i} \frac{d \theta_{j}}{d t}=p_{\Omega}-c_{1} \frac{d \theta_{j}}{d t}
$$

where $p_{\Omega}$ is the price when there is no fluctuation in frequency, the linearized price relations can be described as follows:

$\widehat{P}_{i}(t) \approx P_{i}+c_{2} \cdot\left(p_{i}-p_{\Omega}\right)$.

Hence plugging (7) into (6) we get:

$\widehat{P}_{i}(t) \approx P_{i}-\gamma_{i} \frac{d \theta_{i}(t)}{d t}, \forall i \in\{1, \ldots, N\}$.

As given in [7] that the adaptation is not instantaneous as the response is generally delayed by a time $\tau$ due to measurement and reaction time. Hence, incorporating the economic aspects derived in (8) in (3), the overall equation for the power system dynamics becomes:

$$
\begin{aligned}
& \frac{d^{2} \theta_{j}}{d t^{2}}=P_{j}-\alpha_{j} \frac{d \theta_{j}}{d t}+\sum_{k} K_{j k} \sin \left(\theta_{k}-\theta_{j}\right)+\gamma_{i} \frac{d \theta_{i}(t)}{d t}, \\
& \forall i \in\{1, \ldots, N\} .
\end{aligned}
$$

As given in [6] that instantaneous value of $\theta_{i}(t)$ can cause grid instabilities even for smaller values of $\tau$. Hence, frequency measurements averaged over an intervals of length $T$ is preferred which can stabilize the performance of the grid for wider $\tau$ values. Hence such averaging yields:

$$
\begin{gathered}
\frac{d^{2} \theta_{j}}{d t^{2}}=P_{j}-\alpha_{j} \frac{d \theta_{j}}{d t}+\sum_{k} K_{j k} \sin \left(\theta_{k}-\theta_{j}\right) \\
-\frac{\gamma_{i}}{T} \int_{t-T}^{t} \frac{d \theta_{i}}{d t}\left(t^{\prime}-\tau\right) d t^{\prime} \\
=P_{j}-\alpha_{j} \frac{d \theta_{j}}{d t}+\sum_{k} K_{j k} \sin \left(\theta_{k}-\theta_{j}\right) \\
-\frac{\gamma_{i}}{T}\left(\theta_{j}(t-\tau)-\theta_{j}(t-\tau-T)\right) .
\end{gathered}
$$

Several assumptions have been used in framing the models in Section A and B for the analysis which is described in [8] but the analysis is done considering the heterogeneous response of the consumers. The definition of the stability index for the grid is derived in the following section.

\section{Stability Parameters}

The stability of the dynamical system is found out based on the nature of the eigen values, which is calculated with the help of polynomial calculated from the Jacobian of the system, which is defined in [7] and given as:

$$
J_{\tau}=\left[\begin{array}{cc}
\frac{\partial}{\partial \theta_{\tau, i}}\left(\frac{d}{d t} \theta_{j}\right) & \frac{\partial}{\partial \omega_{\tau, i}}\left(\frac{d}{d t} \theta_{j}\right) \\
\frac{\partial}{\partial \theta_{\tau, i}}\left(\frac{d}{d t} \omega_{j}\right) & \frac{\partial}{\partial \omega_{\tau, i}}\left(\frac{d}{d t} \omega_{j}\right)
\end{array}\right] \in \mathbb{R}^{2 N \times 2 N},
$$

where $\theta_{\tau, i}=\theta_{i}(t-\tau)$ and $\omega_{\tau, i}=\frac{d \theta_{i}}{d t}(t-\tau), i, j \in\{1, \ldots, N\}$.

The characteristic equation of the system for the Jacobian considering the two delays $\tau$ and $\tilde{T}=T+\tau$ is given by: 


$$
p(\lambda)=\operatorname{det}\left(J_{0}+\mathrm{e}^{-\lambda t} J_{\tau}+\mathrm{e}^{-\lambda \tilde{T}} J_{\tilde{T}}-\lambda\right)=0 .
$$

The data set used for analysis is formed with 10,000 randomly chosen initial values for the system defined in (10) and the eigenvalues calculated based on the solutions obtained after solving the polynomial, given in (12). The numerical values of the parameters used in the system is same as the one given in [8].

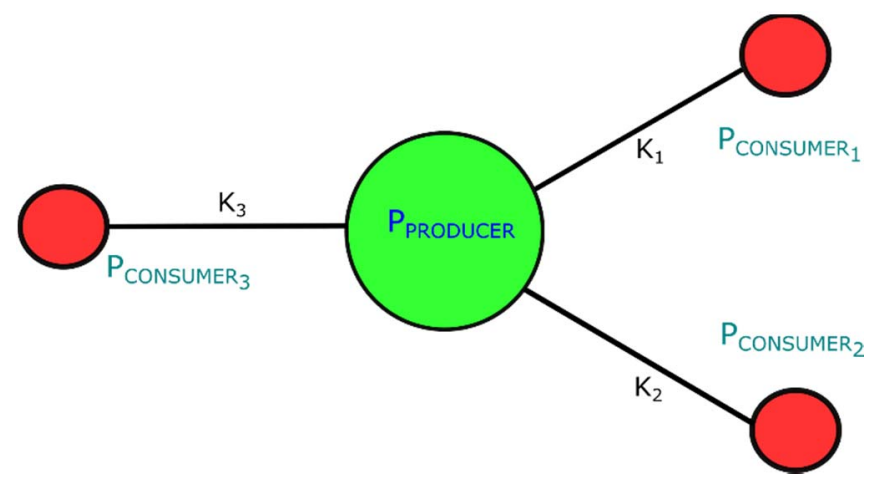

Figure 1: The decentralized control structure.

The stability analysis of the system with decentralized generation is considered for consumers in [7] having similar characteristics which makes the scheme unattractive for heterogeneous consumers as discussed before. Thus, the analysis in this paper has been done based on heterogeneous response of the consumers connected to the producer shown in Figure 1 which is based on the scheme given in [8]. However, the dependence of the input parameters on the grid stability is discussed in this paper using feature selection techniques and Bayesian regression technique. Brief description of the statistical techniques used is given in the following section.

\section{StATISTICAL AND MACHINE LEARNING METHODS}

\section{A. Feature Selection}

Feature selection is a commonly used technique used for model construction as it helps in simplification of models, which can be easily interpreted [20]. It is also helpful in avoiding the curse of dimensionality and it enhances generalization by reducing overfitting [21]. The most commonly used index for feature selection is Fisher score which is based on the selection of features where the distance of the data points, belonging in similar and different classes should be as small and large as possible respectively.

The Fisher score can be computed as follows and given in [22]:

$$
F(\mathbf{Z})=\operatorname{trace}\left\{\widetilde{\mathrm{S}_{\mathrm{b}}}\left(\widetilde{\mathrm{S}_{\mathrm{t}}}+\gamma I\right)^{-1}\right\}
$$

where, $\widetilde{\mathrm{S}_{\mathrm{b}}}$ is the between class scatter matrix and $\widetilde{\mathrm{S}_{t}}$ is the total scatter matrix of the dataset.

Considering the input data matrix as $X \in \mathbb{R}^{d \times n}$ and if $\mu_{k}^{j}$ and $\sigma_{k}^{j}$ be the mean and standard deviation of the $k^{\text {th }}$ class corresponding to the $j^{\text {th }}$ feature. Now consider $\mu^{j}$ and $\sigma^{j}$ be the mean and standard deviation of the whole data set with respect to the $j^{\text {th }}$ feature, the Fisher score can be computed as follows:

$F\left(x^{j}\right)=\frac{\sum_{k=1}^{c} n_{k}\left(\mu_{k}^{j}-\mu^{j}\right)^{2}}{\left(\sigma^{j}\right)^{2}}$,

where, $\left(\sigma^{j}\right)^{2}=\sum_{k=1}^{c} n_{k}\left(\sigma^{j}\right)^{2}$.

Hence based on the value of $F\left(x^{j}\right)$ computed from (14) we can rank the feature influencing the stability of the grid.

\section{B. Regression Analysis}

Regression is a method to model a mathematical relationship between the dependant variables and the response variables. If the relationship is linear in nature, they are called linear models [23], which can be expressed as:

$y_{i}=X_{i} \beta+\epsilon_{i}=\beta_{1} X_{i 1}+\ldots+\beta_{k} X_{i k}+\epsilon_{i}, \quad i \in\{1, \ldots, N\}$.

An equivalent representation of (16) is

$y_{i} \sim \mathcal{N}\left(X_{i} \beta, \sigma^{2}\right), \quad i \in\{1, \ldots, N\}$.

where $X$ is $n$ by $k$ matrix with $i^{\text {th }}$ row $X_{i}$.

Using the multivariate notations, the response variable can be written as:

$y_{i} \sim \mathcal{N}\left(X \beta, \sigma^{2} I\right)$.

Fitting the model using (18) gives an estimate of $\widehat{\beta}$ and $\hat{\sigma}$ from the given data. Standard error in the uncertainty from the coefficient estimates is represented in the form of the estimated covariance matrix $V_{\beta} \hat{\sigma}^{2}$ where the diagonal elements represent the estimated variance of the individual components of $\beta$ and the off-diagonal represents the covariance of estimation.

In order to measure the explained variance in the data from the linear model quantities such as residual standard deviation $\hat{\sigma}$ and explained variation $R^{2}$ is used which can be represented as follows and given in [24]:

$\hat{\sigma}=\sqrt{\sum_{i=1}^{n} r_{i}^{2} /(n-k)}$,

$R^{2}=1-\hat{\sigma}^{2} / s_{y}^{2}$,

where, $n$ and $k$ represents the number of data points and coefficients to be estimated respectively. $s_{y}$ is the standard deviation of the whole data and $r_{i}$ is the residuals of the data which the difference between the data and the fitted values given as follows:

$r_{i}=y_{i}-X_{i} \hat{\beta}$. 
Adjusted $R_{\text {adj }}^{2}$ for the regression problem takes into account the value of explained variation which manages the number of explanatory terms relative to the number of data points [25] which can be described as:

$$
\bar{R}^{2}=1-\left(1-R^{2}\right) \frac{n-1}{n-k-1} .
$$

Above expression can also be written as:

$$
\bar{R}^{2}=1-\frac{\hat{\sigma}^{2} /(n-1)}{s_{y}^{2} /(n-k-1)} .
$$

The $F$-statistic used for the linear regression checks the null hypothesis that whether the test statistic has $F$-distribution under null hypothesis. For regression problem, it checks whether a model which has $p_{1}$ parameters fits the data better than another model having $p_{2}$ parameters. The parameter can be calculated with the help of the mathematical relation given by:

$$
F=\left(\frac{\mathrm{RSS}_{1}-\mathrm{RSS}_{2}}{p_{2}-p_{1}}\right) /\left(\frac{\mathrm{RSS}_{2}}{n-p_{2}}\right)
$$

where, $\mathrm{RSS}_{i}$ is the residual sum of squares for the model $i$.

Quantile plots can be used to check the nature of the residuals obtained after regression [26]. It is generally used to check the distributions of the residuals comparing it with the standard normal distribution. If they are similar then they will fall along the straight line in the plot. They are generally plotted against the quantiles obtained after plotting against the indexes following the normal distribution.

The initial step in the Q-Q- plot is to sort the residuals with increasing size thus $r_{(i)}$ is the $i^{\text {th }}$ smallest residual and hence known as order statistic. Thus in order to compare it with the standard normal distribution, order statistics for the $i^{\text {th }}$ sample is created for the sample size $n$ with the help of the formula expression as described in [27]:

$$
E\left(z_{(i)}\right) \approx \Phi^{-1}[(i-0.3175) /(n+0.365)],
$$

where, $\Phi^{-1}$ is the inverse of the standard normal distribution function.

\section{RESUlts AND Discussions}

The data for the analysis is taken from the UCI Machine Learning Repository database - Electrical Grid Stability Simulated Data [28] where the information regarding the grid stability nature and eigenvalues along with the test parameters are provided. Feature selection is performed on the test parameters namely, response time $\tau$, price response index $\gamma$ and the power produced and consumed $P$. Since the data is based on the configuration given in Figure 1, the power produced is equal to the total consumption.

$P_{1}=P_{2}+P_{3}+P_{4}$
Subscript 1 in equation (26) in the given data belongs to the attribute of the producer while the remaining subscripts belong to the heterogeneous consumers. The features are summarized in TABLE 1.

TABLE 1: Features Data

\begin{tabular}{|l|l|}
\hline Features & Significance \\
\hline $0-3$ & $\tau_{1}, \tau_{2}, \tau_{3}, \tau_{4}$ \\
\hline $4-7$ & $P_{1}, P_{2}, P_{3}, P_{4}$ \\
\hline $8-11$ & $\gamma_{1}, \gamma_{2}, \gamma_{3}, \gamma_{4}$ \\
\hline
\end{tabular}

Thus it is important to identify the features which play an important role in the determining the grid stability. It is done with the help of feature selection described in the previous section where the feature score is determined with the help of (14). The scores for the individual features have been shown in Figure 2.

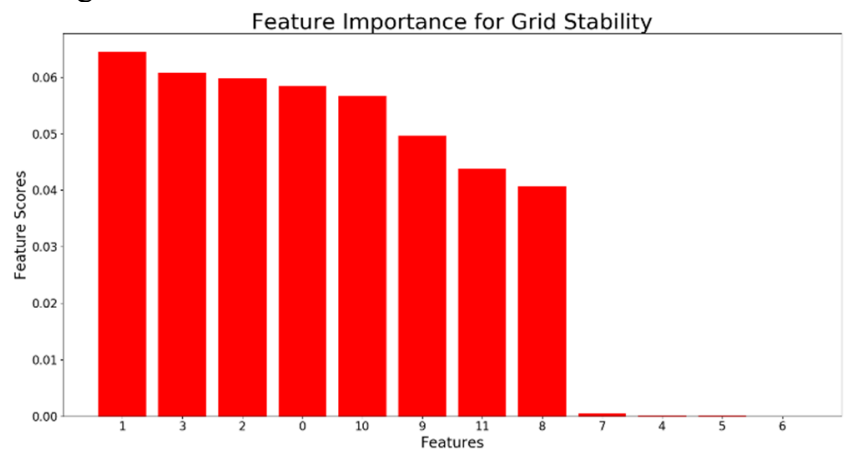

Figure 2: Feature selection results described as sorted feature scores.
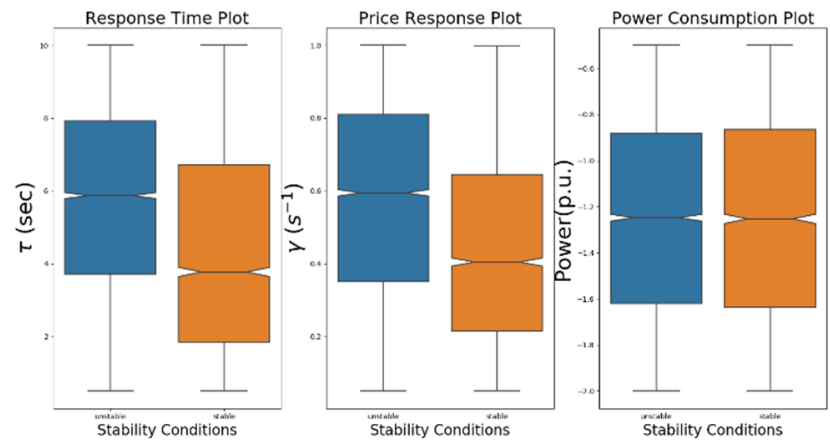

Figure 3: Parameters for consumers under grid stability conditions. Blue: unstable, red: stable condition.

Figure 2 that the power consumption or production by the producer and heterogeneous consumers do not influence the stability of the grid. The time response of the consumers and producer to the price fluctuation play a more important role in the grid stability, as compared to the price elasticity index. The time response and price elasticity of the producer influences the grid stability the least as compared to the parameters of the consumers. This analysis can be confirmed by checking the parameters of the grid during stable and unstable conditions. 
We see in Figure 3 that unstable grid conditions generally prevail for higher values of $\tau$ and $\gamma$. However, the power consumption pattern does not show any difference under stable and unstable grid conditions thus conforming with the analysis of feature selection obtained. Similar plots can be obtained for the producers given in Figure 4, where expected results are found except the power production section when the variability is lower, as compared to the former case.
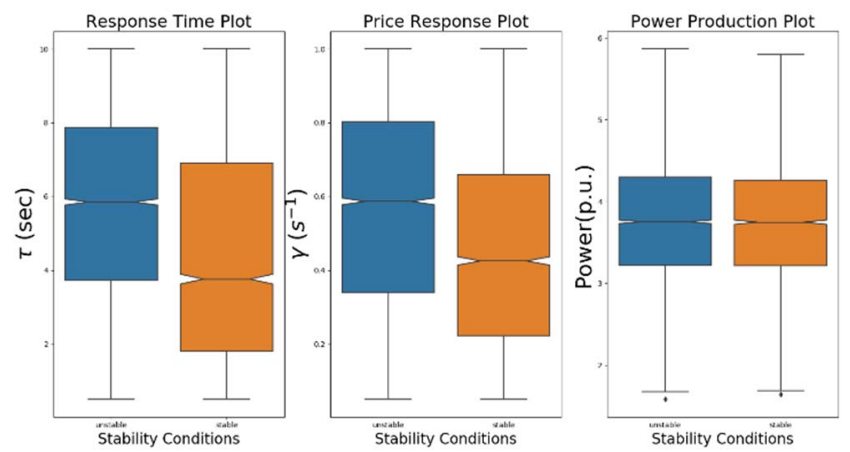

Figure 4: Parameters for producer under grid stability conditions. Blue: unstable, red: stable condition.

Now, regression analysis is performed based on the price response index $\gamma$ and response time $\tau$ for the consumers and the producers relating the eigenvalues of the given system as per the solution of the characteristic equation given in (12). The results of the regression analysis have been shown in Table 2 .

Table 2: Regression Statistics

\begin{tabular}{|l|l|}
\hline Statistic & Value \\
\hline Multiple $R^{2}$ & 0.6468 \\
\hline Adjusted $R^{2}$ & 0.6465 \\
\hline$F$-Statistic & 2287 \\
\hline Degrees of Freedom & 9991 \\
\hline
\end{tabular}

QQ-Plot residuals

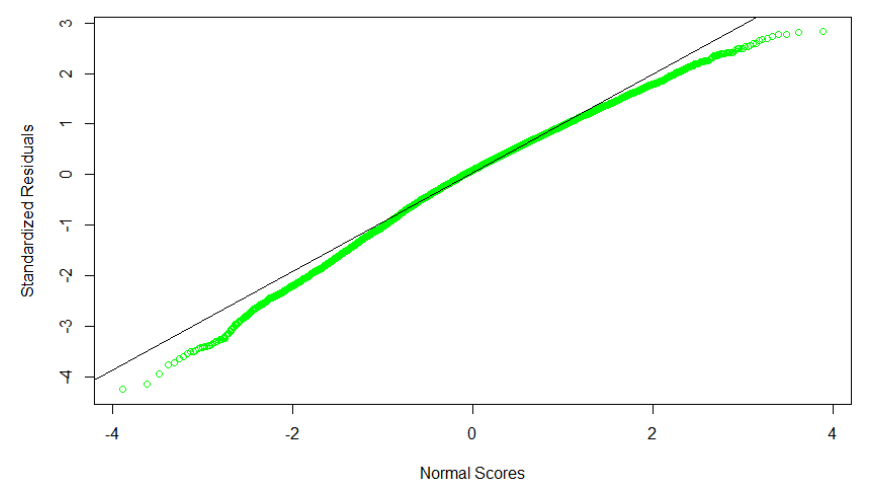

Figure 5 Quantile plot from the regression.

The $R^{2}$ value relates to the fact that linear regression model fitted as per (17) shows decent results in predicting the eigenvalue of the system based on the input parameters. High value of $F$-statistic suggests that intercept only model will fail to describe the data analysed here. The proximity in the values of $R^{2}$ and $\bar{R}^{2}$ suggests that the number of data points are good enough for the predicting the target value.

The quantile plot as shown in the Figure 5 indicate that the plot close to the straight line and hence revealing the distribution of the residuals being close to normal. The coefficients of regression $\hat{\beta}$ and the standard error $\hat{\sigma}$ obtained based on the estimate are shown in Figure 6 .

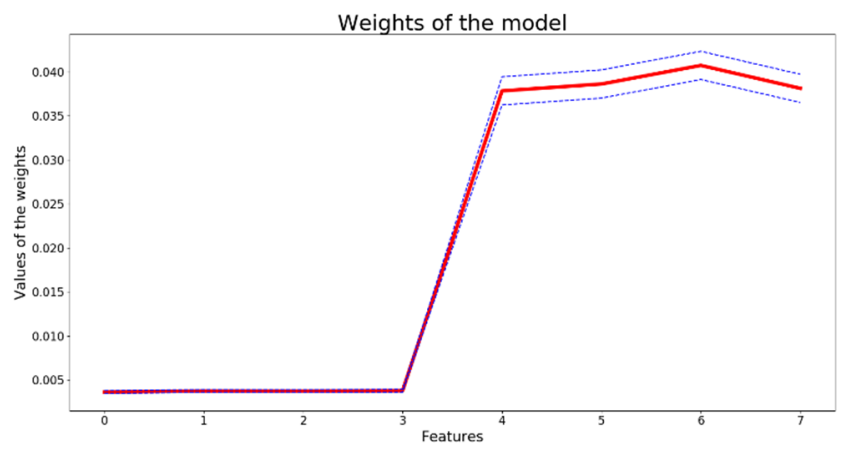

Figure 6 Coefficients of the model variables.

The coefficients suggests that the eigenvalues are more sensitive to the change in the price response index $\gamma\left(X_{8}-X_{11}\right)$ as compared to the response time $\tau\left(X_{0}-X_{3}\right)$. We see from Figure 6 that the estimation error in the coefficients for $\gamma$ is higher as compared to the error in coefficients of $\tau$ which is quite similar for all the consumers and producers. Hence, the regression analysis suggests that the eigenvalues of the system are more sensitive to the change in price response index as compared to the response time of consumers and producer. In addition, the analysis suggests that power production and consumption $\left(X_{4}-X_{7}\right)$ do not have any significant influence on the stability of the grid. Table 2 shows that the $R^{2}$ values are not high enough since the primary goal here is to identify the important variables using linear regression analysis after feature selection. However, owing to the complexity of the power system modelling involved, using higher order kernelized regression models may yield a better fitted model which will be pursued in a future research. Also, effect of other dimensionality reduction and shrinkage methods are also worth exploring in the current context. Cross-validation methods are also known to be very useful to prevent overfitting of complex regression models. We did not explore resampling and cross-validation methods in this paper, since after feature selection, we applied a simple liner regression method as a feasibility study on this synthetic dataset which is less prone to overfitting.

The present regression analysis is shown on a simple grid model as shown in Figure 1 with 1 producer and 3 consumers. The modelling technique described above is generic and can be used to create a more complex synthetic dataset for such regression analysis and validate the findings on variable importance using larger power networks. Analysing this particular dataset can also be viewed as a classification problem, since one might not be interested in predicting the 
eigenvalues, rather intends to know the stability condition only. Also, some areas in the feature space may not be uniformly filled with the random selection of the initial conditions and are sparse in nature. Specific machine learning methods like the Gaussian processes may be useful for such problems that can produce the uncertainties in the regression modelling as a function of the covariates or selected features which will be explored in depth, in our future research.

\section{CONCLUSION}

Decentralized smart grid control has been shown here as a method for demand response. The control strategy has been formulated considering the assumptions, thus finding a deeper insight about the dependence of the parameters of the grid to its stability. The data used for the analysis consists of the response of heterogeneous consumers for different price fluctuations based on the power balance of the grid. The statistical and machine learning methods such as feature selection and regression analysis are performed to find relationship between the input parameters with the eigenvalue and system stability conditions. Future work will dwell up on investigating more complex statistical models for predicting the stability of the grid under more realistic scenarios and complex physical models with decentralized power production systems.

\section{REFERENCES}

[1] S. Grijalva and M. U. Tariq, "Prosumer-based smart grid architecture enables a flat, sustainable electricity industry," in ISGT 2011, 2011, pp. $1-6$.

[2] V. C. Gungor, D. Sahin, T. Kocak, S. Ergut, C. Buccella, C. Cecati, and G. P. Hancke, "Smart grid technologies: Communication technologies and standards," IEEE Transactions on Industrial Informatics, vol. 7, no. 4, pp. 529-539, 2011.

[3] J. Liu, Y. Xiao, S. Li, W. Liang, and C. P. Chen, "Cyber security and privacy issues in smart grids," IEEE Communications Surveys \& Tutorials, vol. 14, no. 4, pp. 981-997, 2012.

[4] M. B. Line, I. A. Tøndel, and M. G. Jaatun, "Cyber security challenges in Smart Grids," in 2011 2nd IEEE PES International Conference and Exhibition on Innovative Smart Grid Technologies, 2011, pp. 1-8.

[5] J. A. Short, D. G. Infield, and L. L. Freris, "Stabilization of grid frequency through dynamic demand control," IEEE Transactions on Power Systems, vol. 22, no. 3, pp. 1284-1293, 2007.

[6] B. Schäfer, M. Matthiae, M. Timme, and D. Witthaut, "Decentral smart grid control," New Journal of Physics, vol. 17, no. 1, p. 015002, 2015.

[7] B. Schäfer, C. Grabow, S. Auer, J. Kurths, D. Witthaut, and M. Timme, "Taming instabilities in power grid networks by decentralized control," The European Physical Journal Special Topics, vol. 225, no. 3, pp. 569-582, 2016.

[8] V. Arzamasov, K. Böhm, and P. Jochem, "Towards Concise Models of Grid Stability," in 2018 IEEE International Conference on Communications, Control, and Computing Technologies for Smart Grids (SmartGridComm), 2018, pp. 1-6.

[9] K. Sajan, V. Kumar, and B. Tyagi, "Genetic algorithm based support vector machine for on-line voltage stability monitoring," International Journal of Electrical Power \& Energy Systems, vol. 73, pp. 200-208, 2015.

[10] V. Malbasa, C. Zheng, P.-C. Chen, T. Popovic, and M. Kezunovic, "Voltage stability prediction using active machine learning," IEEE Transactions on Smart Grid, vol. 8, no. 6, pp. 3117-3124, 2017.

[11] J. Hazra, R. K. Reddi, K. Das, D. P. Seetharam, and A. K. Sinha, "Power grid transient stability prediction using wide area synchrophasor measurements," in $20123 r$ IEEE PES Innovative Smart Grid Technologies Europe (ISGT Europe), 2012, pp. 1-8.
[12] B. Wang, B. Fang, Y. Wang, H. Liu, and Y. Liu, "Power system transient stability assessment based on big data and the core vector machine," IEEE Transactions on Smart Grid, vol. 7, no. 5, pp. 25612570, 2016.

[13] L. Moulin, A. A. Da Silva, M. El-Sharkawi, and R. J. Marks, "Support vector machines for transient stability analysis of largescale power systems," IEEE Transactions on Power Systems, vol. 19, no. 2, pp. 818-825, 2004.

[14] B. Jayasekara and U. D. Annakkage, "Derivation of an accurate polynomial representation of the transient stability boundary," IEEE Transactions on Power Systems, vol. 21, no. 4, pp. 1856-1863, 2006.

[15] T. Amraee and S. Ranjbar, "Transient instability prediction using decision tree technique," IEEE Transactions on Power Systems, vol. 28, no. 3, pp. 3028-3037, 2013.

[16] Y. Xu, Z. Dong, K. Meng, R. Zhang, and K. Wong, "Real-time transient stability assessment model using extreme learning machine," IET Generation, Transmission \& Distribution, vol. 5, no. 3, pp. 314-322, 2011.

[17] N. Bassamzadeh and R. Ghanem, "Multiscale stochastic prediction of electricity demand in smart grids using Bayesian networks," Applied Energy, vol. 193, pp. 369-380, 2017.

[18] D. Yang, L. Xu, S. Gong, H. Li, G. D. Peterson, and Z. Zhang, "Joint electrical load modeling and forecasting based on sparse bayesian learning for the smart grid," in 2011 45th Annual Conference on Information Sciences and Systems, 2011, pp. 1-6.

[19] G. Filatrella, A. H. Nielsen, and N. F. Pedersen, "Analysis of a power grid using a Kuramoto-like model," The European Physical Journal $B$, vol. 61, no. 4, pp. 485-491, 2008.

[20] G. James, D. Witten, T. Hastie, and R. Tibshirani, An introduction to statistical learning, vol. 112. Springer, 2013.

[21] M. L. Bermingham, R. Pong-Wong, A. Spiliopoulou, C. Hayward, I. Rudan, H. Campbell, A. F. Wright, J. F. Wilson, F. Agakov, P. Navarro, and others, "Application of high-dimensional feature selection: evaluation for genomic prediction in man," Scientific Reports, vol. 5, p. 10312, 2015.

[22] Q. Gu, Z. Li, and J. Han, "Generalized fisher score for feature selection," In Proceedings of the Twenty-Seventh Conference on Uncertainty in Artificial Intelligence (pp. 266-273). AUAI Press, 2011.

[23] H. L. Seal, The historical development of the Gauss linear model. Yale University New Haven, 1968.

[24] J. Neter, M. H. Kutner, C. J. Nachtsheim, and W. Wasserman, Applied linear statistical models, vol. 4. Irwin Chicago, 1996.

[25] J. J. Faraway, Linear models with R. Chapman and Hall/CRC, 2016.

[26] M. C. Wang and B. J. Bushman, "Using the normal quantile plot to explore meta-analytic data sets.," Psychological Methods, vol. 3, no. 1, p. 46, 1998.

[27] J. J. Filliben, "The probability plot correlation coefficient test for normality," Technometrics, vol. 17, no. 1, pp. 111-117, 1975.

[28] "Electrical Grid Stability Simulated DataSet" [Online]. Available: https://archive.ics.uci.edu/ml/datasets/Electrical+Grid+Stability+Si mulated + Data + . 